\title{
A novel dual bandpass filter incorporating left-handed transmission line based dual-band resonator
}

\author{
Xin Wang ${ }^{1}$, Hsien-Shun $\mathrm{Wu}^{1 \mathrm{a})}$, and Ching-Kuang Tzuang ${ }^{2}$ \\ ${ }^{1}$ School of Electronic Information Engineering, Tianjin University, \\ Tianjin 300072, People's Republic of China \\ ${ }^{2}$ Millimeter-wave and Terahertz Technologies Transcend Research Center, \\ Beijin 100049, People's Republic of China
}

a)seanwu@ieee.org

\begin{abstract}
This paper presents a left-handed transmission line (LH TL) based dual-band resonator to implement the dual-band filter. The proposed resonator consists of one uniform LH TL and one right-handed transmission line (RH TL) to produce three transmission zeros at $52 \mathrm{GHz}, 267 \mathrm{GHz}$, and $531 \mathrm{GHz}$. The perforated ground plane is applied to extend the operating bandwidth of the LH TL for covering the full bandwidth of the dual-band filter. A CMOS prototype is designed based on the proposed resonator, and measured through the on-wafer measurements. Both simulated and the measured results confirm the insertion-losses of $1.8 \mathrm{~dB}$ and $2.4 \mathrm{~dB}$ of two passbands at $186 \mathrm{GHz}$ and $420 \mathrm{GHz}$. The statistic shows the total length of the proposed resonator is about $0.038 \lambda_{\mathrm{g}}$ at $52 \mathrm{GHz}$.
\end{abstract}

Keywords: Bandpass filter, dual-band, left-handed transmission line (LH TL), dual-band resonator, CMOS

Classification: Microwave and millimeter wave devices, circuits, and systems

\section{References}

[1] C. Y. Chen and C. Y. Hsu: IEEE Microw. Wireless Compon. Lett. 16 (2006) 246. DOI:10.1109/LMWC.2006.873584

[2] L. C. Tsai and C. W. Hsue: IEEE Trans. Microw. Theory Techn. 52 (2004) 1111. DOI:10.1109/TMTT.2004.825680

[3] J. T. Kuo, T. H. Yeh and C. C. Yeh: IEEE Trans. Microw. Theory Techn. 53 (2005) 1331. DOI:10.1109/TMTT.2005.845765

[4] C. Quendo, A. Manchec, Y. Clavet, E. Rius, J.-F. Favennec and C. Person: IEEE Microw. Wireless Compon. Lett. 17 (2007) 337. DOI:10.1109/LMWC. 2007.895697

[5] C. Quendo, E. Rius and C. Person: IEEE MTT-S Int. Microw. Symp. Dig. 2 (2003) 1093. DOI:10.1109/MWSYM.2003.1212559

[6] C.-Z. Hua, C. Miao and W. Wu: Asia Pacific Microwave Conference (2009) 1383. DOI:10.1109/APMC.2009.5384494

[7] L. K. Yeh, C. Y. Hsu, C. Y. Chen and H. R. Chuang: IEEE Electron Device Lett. 29 (2008) 1373. DOI:10.1109/LED.2008.2006696 
[8] C. Quendo, E. Rius and C. Person: IEEE Trans. Microw. Theory Techn. 51 (2003) 734. DOI:10.1109/TMTT.2003.808729

[9] C. C. Chen and C.-K. C. Tuang: IEEE Trans. Microw. Theory Techn. 52 (2004) 1637. DOI:10.1109/TMTT.2004.828468

[10] A. Lai, C. Caloz and T. Itoh: IEEE Microw. Mag. 5 (2004) 34. DOI:10.1109/ MMW.2004.1337766

[11] M. J. Chiang, H. S. Wu and C.-K. C. Tzuang: IEEE Trans. Microw. Theory Techn. 55 (2007) 2512. DOI:10.1109/TMTT.2007.910089

[12] X. R. Li, H. S. Wu and C.-K. C. Tzuang: IEEE Int. Wireless Symp. (2014) 1. DOI:10.1109/IEEE-IWS.2014.6864261

[13] H.-S. Wu, H.-J. Yang, C.-J. Peng and C.-K. C. Tzuang: IEEE Trans. Microw. Theory Techn. 53 (2005) 2713. DOI:10.1109/TMTT.2005.854193

[14] G. L. Matthaei, L. Young and E. M. T. Jones: Microwave Filters, ImpedanceMatching Networks, and Coupling Structures (Artech House, Dedham, MA, 1980) 100.

\section{Introduction}

The dual-band bandpass filter (DBPF) has been gaining wide attention. The dual passband responses can be realized by the multiple-filter topology, including two bandpass filters (BPF) in the parallel connection [1], and a BPF cascading a bandstop filter (BSF) [2]. The multiple-filter implementation needs to the additional wideband impedance matching network to minimize the impedance mismatch between two different filters. In the single-filter architecture, the parallel-coupled microstrip BPF uses the stepped impedance resonator (SIR) to control the multiple resonances for synthesizing the second passband [3]. On the other hand, the $N$-band resonator consists of multiple open-ended shunt stubs to produce multiple transmission zeros for making multiple passbands [4]. For example, a single filter consisting of two dual-band resonators can realize dual second-order bandpass responses $[5,6]$. In the conventional dual-band resonator, the transmission zero at lower frequency requires the shunt stub with longer physical length [5]. The wider frequency-spacing of two passbands requires higher characteristic-impedance ratio in the non-uniform shunt stubs [6,7]. This paper uses a uniform left-handed transmission line (LH TL) and right-handed transmission line to miniaturize the dual-band resonator. A CMOS prototype in a chip area of $128 \mathrm{um} \times 99 \mathrm{um}$ achieves the second-order and third-order bandpass responses at $180 \mathrm{GHz}$ and $420 \mathrm{GHz}$ with $32 \%$ and $16 \%$ bandwidth, respectively. The statistic shows that the proposed dualband resonator has a total length of $0.0318 \lambda_{\mathrm{g}}$ at $52 \mathrm{GHz}$, achieving ten-time shorter than the reported resonators in $[5,6]$.

\section{Dual-band filter incorporating left-handed transmission line based dual-band resonator}

Fig. 1(a) shows the left-handed transmission line (LH TL) based dual-band resonator. Three transmission zeros, including $f_{\mathrm{z} 1}, f_{\mathrm{z} 2}$, and $f_{\mathrm{z} 3}$, realize two passbands at $f_{\mathrm{c} 1}$, and $f_{\mathrm{c} 2}$. The right-handed transmission line (RH TL), and LH TL, which are marked by the black and grey inks, are quarter-wavelength long at 


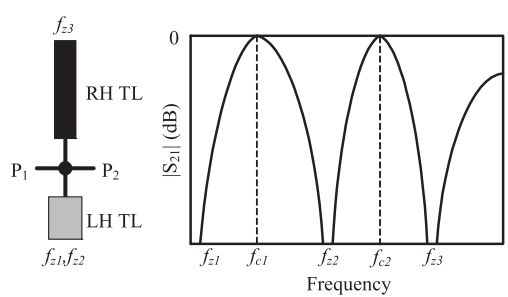

(a)

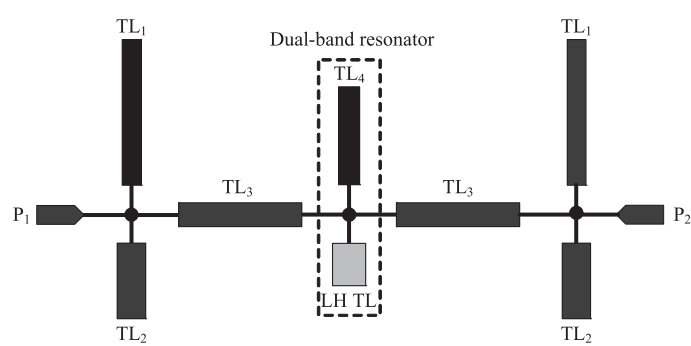

(b)

Fig. 1. Dual-band bandpass filter. (a): Left-handed transmission line (LH TL) based dual-band resonator, (b) Schematic of the dualband filter. The parameters of the elements are shown in Fig. 4.

the frequencies at $f_{\mathrm{z} 2}$, and $f_{\mathrm{z} 3}$, respectively. Below the cut-off frequency of the LH $\mathrm{TL}$, the evanesced mode in the LH TL produces the lowest transmission zero at $f_{\mathrm{z} 1}$. The proposed architecture use one uniform LH TL to control two transmission zeros of the dual-band resonator, making the resonator miniaturization. Fig. 1(b) indicates the schematic of the dual-band filter constructed by the proposed dualband resonator in Fig. 1(a). All the element with black inks are the RH TLs. TL and $\mathrm{TL}_{2}$ form a conventional dual behavior resonator [8], contributing additional two transmission zeros to enhance the spectrum rejections between two passbands and above the second passband. Consequently, the proposed dual-band resonator, incorporating with two $\mathrm{TL}_{1}$ and $\mathrm{TL}_{2}$, constructs second passband in the order of three. $\mathrm{TL}_{3}$ acts as the impedance inverter in the second passband to control the adjacent coupling of the parallel resonators. In the first passband, by combing with $\mathrm{TL}_{1}$, the dual-band resonator makes the bandpass response in the order of two. As shown in Fig. 1, all the RH TL can be implemented by the complementaryconducting-strip transmission line (CCS TL) [9]. The CCS TL, defined by five structural parameters, can realize the guiding characteristics for the filter design, and make the structure in the meandered form for the compact design.

The dual-band filter implementation shown in Fig. 1 is based on one major assumption. The LH TL must achieve a negative phase constant in the bandwidth covering the entire dual passbands. This assumption demands the LH TL syntheses with wide bandwidth. To accomplish the wideband LH TL design, Fig. 2 presents the unit cell of LH TL based on the standard $0.13 \mu \mathrm{m}$ CMOS 1P8M technology. The unit cell, which consists of a metal-oxide-metal (MOM) capacitance and a grounded spiral inductor, is shielded by the perforated ground. The perforated ground, instead of the uniform ground, can effectively reduce the shunt capacitances $\left(C_{R}\right)$ in the unit cell, extending the highest frequency of the left-handed region of the composite right/left-handed (CRLH) transmission line [10]. The size of the unit cell, defined by $\mathrm{P}$, is $14.18 \mu \mathrm{m}$. The metal layers M1 and M4, connected by the VIAs, form the mesh ground plane. The mesh area, defined by $\mathrm{W}_{\mathrm{h}}$, is $13.36 \mu \mathrm{m}$. In the MOM capacitor, the finger width $\left(\mathrm{W}_{\mathrm{C}}\right)$, length $\left(\mathrm{L}_{\mathrm{C}}\right)$, and spacing $\left(\mathrm{S}_{\mathrm{C}}\right)$ are $13 \mu \mathrm{m}, 9.68 \mu \mathrm{m}$, and $0.5 \mu \mathrm{m}$. The line width $\left(\mathrm{W}_{\mathrm{L}}\right)$ and lengths $\left(\mathrm{L}_{1}\right.$ and $\left.\mathrm{L}_{2}\right)$ in the spiral inductor are $2.5 \mu \mathrm{m}, 5.54 \mu \mathrm{m}$, and $6.56 \mu \mathrm{m}$. The parameters in the equivalent model of the unit cell, including $\mathrm{C}_{\mathrm{R}}, \mathrm{L}_{\mathrm{R}}, \mathrm{C}_{\mathrm{L}}$, and $\mathrm{L}_{\mathrm{L}}$, are $8 \mathrm{fF}, 3 \mathrm{pH}$, $21 \mathrm{fF}, 11 \mathrm{pH}$. 


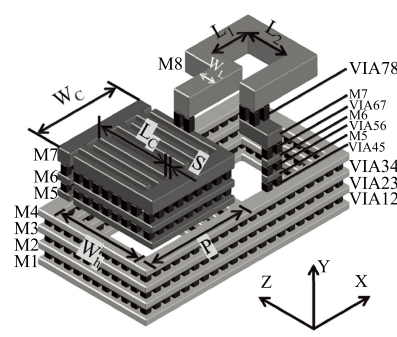

(a)

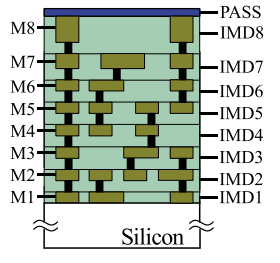

(b)

Fig. 2. Unit cell of the LH TL based on $0.13 \mu \mathrm{m}$ 1P8M CMOS (a) unit cell (b) cross section of the $0.13 \mu \mathrm{m} 1 \mathrm{P} 8 \mathrm{M}$ CMOS process.

The guiding characteristics of LH TL are extracted from the two-port scattering parameters collected by performing the full-wave electromagnetic (EM) simulation. In the EM simulation, all the material parameters are set as identical to those in [11]. The propagation constant of the LH TL is extracted from ABCD matrix of Ncell LH TL [12]. In this paper, $\mathrm{N}$ is set as fifteen. Fig. 3 shows the extracted results of the LH TL constructed by the unit cell in Fig. 2.

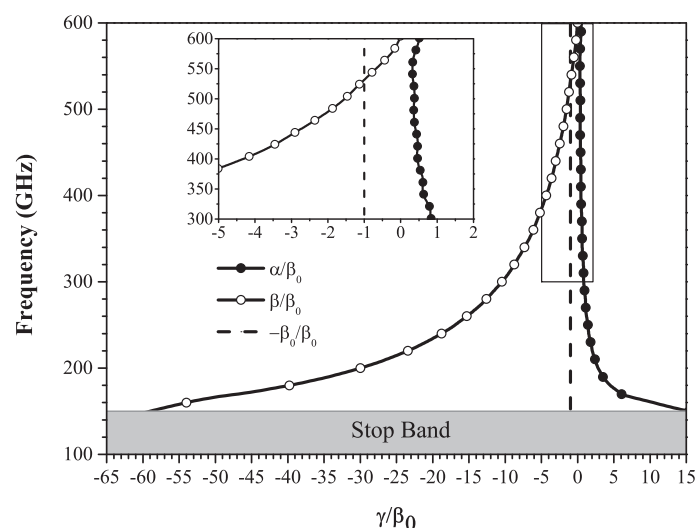

Fig. 3. The complex propagation constant of LH TL based on the unit cell in Fig. 2.

The normalize phase constant $\left(\beta / \beta_{0}\right)$, indicated by the curve with hollow symbol, is defined as the slow wave factor (SWF) of the CMOS LH TL in this paper. As shown in Fig. 3, the cutoff frequency of the LH TL is about $150 \mathrm{GHz}$. SWF is equal to one and zero at $530 \mathrm{GHz}$, and $600 \mathrm{GHz}$, respectively. The operating bandwidth of the CMOS LH TL is about $380 \mathrm{GHz}$. In the next section, a filter prototype based on the proposed dual-band resonator is presented.

\section{CMOS dual-band filter prototype}

To design a dual-band filter based on the concept shown in Fig. 1, two center frequencies $\left(f_{\mathrm{c} 1}\right.$, and $\left.f_{\mathrm{c} 2}\right)$ of the dual passbands are set at $180 \mathrm{GHz}$ and $420 \mathrm{GHz}$, respectively. $f_{\mathrm{z} 1}, f_{\mathrm{z} 2}$, and $f_{\mathrm{z} 3}$ produced by the $\mathrm{TL}_{4}$, and LH TL are set at $52 \mathrm{GHz}$, $267 \mathrm{GHz}$, and $531 \mathrm{GHz}$. The transmission zeros contributed by $\mathrm{TL}_{1}$, and $\mathrm{TL}_{2}$ are set at $315 \mathrm{GHz}$, and $664 \mathrm{GHz}$, respectively. During the dual-passband design, the thirdorder bandpass response at $420 \mathrm{GHz}$ is conducted by following the procedures documented in the Section III of [13] with the Chebyshev responses in the ripple of 
$0.1 \mathrm{~dB}$ [14] before the second-order passband design at $180 \mathrm{GHz}$. As mentioned in Section 2, both $\mathrm{TL}_{1}$, and $\mathrm{LH}$ TL makes the parallel resonances with the other elements at two passbands. $\mathrm{TL}_{3}$ is applied to control the resonators' coupling at two passbands. Therefore, after several design iterations, the values of all the elements can be calculated to support dual passband.

Fig. 4 shows the CMOS prototype with the design parameters of all the elements in Fig. 1(b). The signal trace of all the CCS TLs is realized by M8 and the mesh ground is implemented by stacked M1 to M5. The period of unit cell for CCS TL is $14.18 \mu \mathrm{m}$. The signal width $(\mathrm{W}) /$ mesh area $\left(\mathrm{W}_{\mathrm{h}}\right)$ of $\mathrm{TL}_{1}$ to $\mathrm{TL}_{4}$ are $2.5 \mu \mathrm{m} / 13.36 \mu \mathrm{m}, 5.8 \mu \mathrm{m} / 11.36 \mu \mathrm{m}, 5.9 \mu \mathrm{m} / 11.36 \mu \mathrm{m}, 2.5 \mu \mathrm{m} / 11.36 \mu \mathrm{m} . \mathrm{TL}_{1}$, $\mathrm{TL}_{2}$, and $\mathrm{TL}_{4}$ are in the meandered forms to make filter miniaturization. $\mathrm{LH} \mathrm{TL}$ in the central position of the filter has two unit cells implemented by the structural parameters identical to those of shown in Fig. 2. As shown in Fig. 3, the SWF of LH TL is about 14, establishing 90 degrees phase lead at $267 \mathrm{GHz}$.

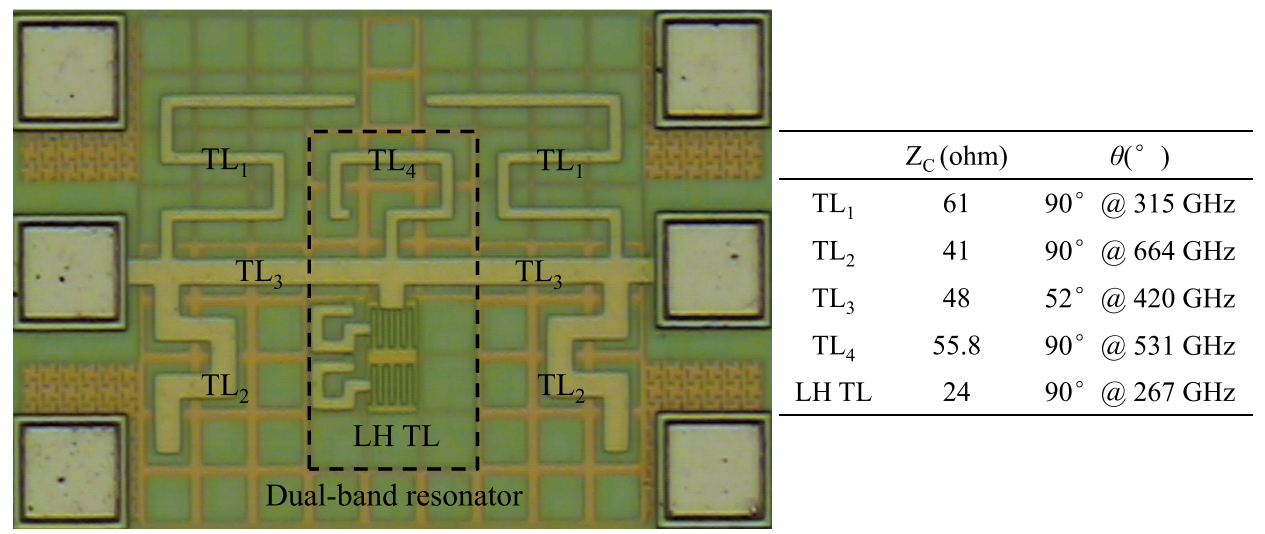

Fig. 4. Prototype of $180 / 420 \mathrm{GHz}$ dual-band filter in CMOS $0.13 \mathrm{um}$ 1P8M technology. The chip size is $128 \mu \mathrm{m} \times 99 \mu \mathrm{m}$.

Before making the on-wafer characterizations, the whole system, including the vector network analyzer, Rohde\&Schwarz ZVA40, with the millimeter frequency converters, ZVA220 (140-220 GHz), ZVA500 (325-500 GHz), cable and probes are calibrated by performing two-port Line-Reflection-Reflection-Match (LRRM) procedures with standard substrates from Cascade Microtech ${ }^{\mathrm{TM}}$. Fig. 5 shows the simulated and measured two-port scattering parameters. The solid curve represents the scattering parameters of the filter by performing the full wave EM simulation with commercial software ANSYSTM HFSS Ver. 15.0. The measured results indicated by the dash line, reveal excellent agreement with the HFSS simulations from $140 \mathrm{GHz}$ to $220 \mathrm{GHz}$ and $325 \mathrm{GHz}$ to $500 \mathrm{GHz}$. The comparison show that the dual-band filter has a $1.8 \mathrm{~dB}$ insertion-loss with $32 \%$ bandwidth at $186 \mathrm{GHz}$ and $2.4 \mathrm{~dB}$ insertion loss with $16 \%$ bandwidth at $420 \mathrm{GHz}$. The return losses are below $-17 \mathrm{~dB}$ in two passbands. As reported in Section 2, the operating bandwidth of the LH TL is from $150 \mathrm{GHz}$ to $530 \mathrm{GHz}$. The leakage phenomena of LH TL above $530 \mathrm{GHz}$ limit the filter rejection of $38 \mathrm{~dB}$ at $540 \mathrm{GHz}, 10 \mathrm{~dB}$ lower than the rejections between $300 \mathrm{GHz}$ and $340 \mathrm{GHz}$. Although the LH TL becomes a right-handed transmission line above $600 \mathrm{GHz}$, the HFSS simulations show that the design can still achieve a $32 \mathrm{~dB}$ rejection from $600 \mathrm{GHz}$ to $700 \mathrm{GHz}$. 


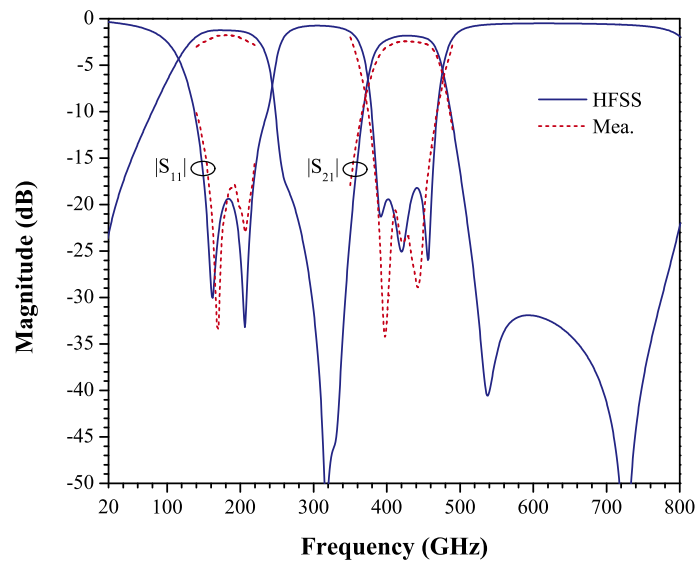

Fig. 5. Comparisons between the simulated and measured two-port scattering parameters of the dual-band bandpass filter.

The curves in Fig. 5 prove the feasibility of the filter implementation based on the proposed dual-band resonator shown in Fig. 1. Table I summarizes the reported dual-band resonator. The total lengths, which are the summations of all the physical lengths of the transmission lines in the resonators, are normalized by the guiding wavelengths at the frequency of the lowest transmission zero for the comparison. The proposed resonator achieves a total length of $0.038 \lambda_{\mathrm{g}}$ at $52 \mathrm{GHz}$, ten-time shorter than the reported resonators.

Table I. Dual-band resonators

\begin{tabular}{ccccc}
\hline Ref. & $\varepsilon_{\mathrm{r}}$ & $f_{z}(\mathrm{GHz})$ & Total length $(\mathrm{mm})$ & Normalized $\left(\lambda_{\mathrm{g}} @ f_{\mathrm{z} 1}\right)$ \\
\hline$[5]$ & 10 & $0.7,1.25,2.1$ & 77 & $0.458 \lambda_{\mathrm{g}}$ \\
{$[6]$} & 2.2 & $3.5,6.5,11.5$ & 22.24 & $0.356 \lambda_{\mathrm{g}}$ \\
This work & 4 & $52,267,531$ & 0.111 & $0.038 \lambda_{\mathrm{g}}$ \\
\hline
\end{tabular}

\section{Conclusions}

A dual-band filter implementation based on the novel dual-band resonator is presented. The proposed resonator consists of one uniform right-handed transmission line (RH TL) and one LH TL to produce three transmission zeros. To extend the operating bandwidth of the LH TL for covering the full spectrum of the dual-band filter, the perforated ground plane is beneath the unit cell to minimize the shut capacitances. The measured results of a CMOS prototype confirm the dual bandpass responses of the filter design based on the proposed resonator. The simulations indicate that the leakage phenomena of LH TL slightly degrade the filter rejection of $10 \mathrm{~dB}$ above $530 \mathrm{GHz}$. The statistic based on the published works show that the proposed resonator achieves the highest circuit miniaturization.

\section{Acknowledgments}

The authors wish to extend their sincere thanks to the Silterra Corporation, Malaysia, for providing the chip fabrication. This work was supported in part by the National Natural Science Foundation of China under Grant 61271068, the 863 Program of China under Grant No. 2015AA01A703, and the Global Expert Program in China. 